\title{
Angular melting scenarios in binary dusty-plasma Coulomb balls: Magic versus normal clusters
}

\author{
S. W. S. Apolinario* and J. Albino Aguiar ${ }^{\dagger}$ \\ Departamento de Física, Universidade Federal de Pernambuco, 50670-901 Recife, PE, Brazil \\ F. M. Peeters ${ }^{\ddagger}$ \\ Departement Fysica, Universiteit Antwerpen, Groenenborgerlaan 171, B-2020 Antwerpen, Belgium
}

(Received 2 June 2014; published 29 December 2014)

\begin{abstract}
Molecular-dynamic simulations were performed in order to investigate the melting processes of isotropically confined binary systems. We considered two species of particles, which differ by their amount of electric charge. A Lindemann type of criterion was used to determine the angular melting temperature. We demonstrate that the magic-to-normal cluster transition can evolve in two distinct ways, that is, through a structural phase transition of the first order or via a smooth transition where an increase of the shells' width leads to a continuous decreasing mechanical stability of the system. Moreover, for large systems, we demonstrate that the internal cluster exerts a minor effect on the mechanical stability of the external shell. Furthermore, we show that highly symmetric configurations, such as those found for multiple ring structures, have large mechanical stability, i.e., high angular melting temperature.
\end{abstract}

DOI: 10.1103/PhysRevE.90.063113

PACS number(s): 52.27.-h, 64.60.-i, 64.70.pv

\section{INTRODUCTION}

During the past years several efforts have been made to understand the static and dynamic properties of strongly coupled systems of interacting particles in external traps. A few examples include ions in Penning and Paul traps [1,2], electrons in quantum dots [3], electrons on the surface of liquid helium [4], colloidal particles [5], mesoscopic charges of dusty plasmas [6,7], and vortices in mesoscopic shaped superconductors [8,9].

Complex plasmas have been typically generated in parallel plate radio-frequency (rf) discharge. Large particles, called dust particles, introduced into the plasma chamber become electrically charged. The effect of the downward gravitational force acting on the dust particles is compensated by a strong upward electric field generated by the lower electrode. Consequently, the dust particles are levitated and form nearly two-dimensional crystals having similarities to colloidal suspensions $[10,11]$ and show a rich set of interesting phenomena, like phase transitions [12,13], fluid motion [14], and a variety of wave phenomena [15].

Gravitational effects are circumvented in experiments under microgravity conditions in space $[16,17]$ or on parabolic flights $[18,19]$. In these cases, extended three-dimensional complex plasmas could be generated. However, the dust is not evenly distributed in the complete sample, but is disturbed by a dust-free region in the center of the discharge, the so-called void phenomenon.

Spherical three-dimensional homogeneous clusters of dust plasma have been recently realized experimentally [20], where a thermophoretic force was applied to compensate for gravity, which in combination with the plasma induced electric-field force establishes a vertical confinement. Such systems were named "Coulomb balls." Theoretical investigation of structural

\footnotetext{
*sergiowsa@df.ufpe.br

†albino@df.ufpe.br

${ }^{\ddagger}$ francois.peeters@ua.ac.be
}

properties and melting behavior in three-dimensional (3D) Coulomb balls are reported in Refs. [21-25]. It was found that inside a Coulomb ball the dust particles are arranged in nested shells. Within each shell the particles form a hexagonal lattice on a sphere, which is affected by defects as a consequence of the curvature of the shell surface. The large size of the dust particles allows direct observation by simple video microscopy because dynamical processes occur on typical frequency scales of a few hertz, quite unlike strongly coupled colloidal suspensions [5], where particle motion is heavily damped. Therefore, this system is ideally suited for studying the static and dynamics of strongly coupled matter with "atomic resolution."

The ground-state (GS) configurations and the lowestenergy metastable states for systems varying from $N=4$ up to 100 particles were determined in our previous work [24]. In the latter, the energy minimization was performed via a Monte Carlo process associated with the optimized Newton minimization algorithm [26]. Physical quantities such as the second derivative of the binding energy and the lowest-energy eigenfrequencies were computed in order to indicate possible candidates to hold a large mechanical stability, that is, to be a magic cluster. As demonstrated from Fig. 10 of Ref. [24] the systems with $N=4,6,10,12,19,32,38,56,81$, and 94 were classified as candidates to be magic clusters, since their second derivative of the binding energy had relatively large values. However, in order to be sure that these configurations are indeed magic clusters, a direct test of the mechanical stability still needed to be done. This requirement was met by Ref. [25], which demonstrated via the use of molecular-dynamics simulation that only the clusters with $N=6,12,13$, and 38 could hold large mechanical stability, and therefore be classified as magic clusters. In this same work, it was also verified that the structure of clusters with more than $N=38$ particles have a relatively large number of defects and usually present non-well-defined concentric shells. In addition, such deformations were shown to be responsible for reducing the mechanical stability of monodisperse systems. 
In this paper we perform molecular-dynamics simulation to probe directly the magic cluster properties and the mechanical stability of 3D isotropically confined binary Coulomb clusters. Such a system is formed by two species of particles, namely particles $A$ and $B$, which differ from each other by their number of charges, i.e., $Q_{A}$ and $Q_{B}$, respectively. The physical properties of the system are investigated as a function of the charge ratio parameter $\beta=Q_{B} / Q_{A}$, and $N_{A}$ and $N_{B}$, that is, the number of particles of $A$ and $B$ type, respectively. We have found that, as the parameter $\beta$ is decreased from unity, the $B$ type particles migrate towards the internal region of the cluster, and when this occurs smoothly, it does not destroy the magic cluster properties of the system. On the other hand, when the reduction of the $\beta$ parameter leads to an abrupt configurational change, which is mediated by a first-order structural phase transition, a significant decrease of the mechanical stability is found. Differently from Ref. [27], where we focused on the low-temperature behavior, here we will perform full molecular-dynamics simulations which are able to reveal new features. For instance, this work shows that an unusually high mechanical stability is present when the GS configuration of the binary system is organized in a multiple ring structure [28,29]. Moreover, it is also demonstrated that the magic cluster property of the binary system is very robust and that it only suffers minor effects from the width of the cluster's shell.

This paper is organized as follows. In Sec. II, the physical model and the numerical approaches are presented. In Secs. III and IV we present our results for systems with small and large sizes, i.e., systems with one and two shells, respectively. Finally, in Sec. V we present our conclusions.

\section{THEORETICAL METHOD}

We study a 3D model system of $N$ charged particles that are confined by an isotropic parabolic potential interacting through a repulsive Coulomb potential. We consider two species of particles $A$ and $B$ having charge $Q_{A}$ and $Q_{B}$, respectively. The potential energy of the system is given by

$$
\begin{aligned}
E= & \sum_{i=1}^{N} \frac{1}{2} m \omega_{0}^{2}\left(x_{i}^{2}+y_{i}^{2}+z_{i}^{2}\right)+\sum_{i>j=1}^{N_{A}} \frac{Q_{A}^{2}}{\epsilon_{0}} \frac{1}{\left|\mathbf{r}_{i}-\mathbf{r}_{j}\right|} \\
& +\sum_{k>l=1}^{N_{B}} \frac{Q_{B}^{2}}{\epsilon_{0}} \frac{1}{\left|\mathbf{r}_{i}-\mathbf{r}_{j}\right|}+\sum_{m=1}^{N_{A}} \sum_{n=1}^{N_{B}} \frac{Q_{A} Q_{B}}{\epsilon_{0}} \frac{1}{\left|\mathbf{r}_{m}-\mathbf{r}_{n}\right|}
\end{aligned}
$$

where $\epsilon_{0}$ and $m$ are, respectively, the dielectric constant and the particle mass, $\mathbf{r}_{i}=\left(x_{i}, y_{i}, z_{i}\right)$ is the coordinate of the $i$ th particle, $N_{A}$ and $N_{B}$ are the total number of particles of type $A$ and $B$, respectively, $N=N_{A}+N_{B}$ is the total number of particles, and $\omega_{0}$ is the confinement frequency. We can rewrite the potential energy (1) in the following dimensionless form:

$$
\begin{aligned}
E= & \sum_{i=1}^{N}\left(x_{i}^{2}+y_{i}^{2}+z_{i}^{2}\right)+\sum_{i>j=1}^{N_{A}} \frac{1}{\left|\mathbf{r}_{i}-\mathbf{r}_{j}\right|} \\
& +\sum_{k>l=1}^{N_{B}} \frac{\beta^{2}}{\left|\mathbf{r}_{i}-\mathbf{r}_{j}\right|}+\sum_{m=1}^{N_{A}} \sum_{n=1}^{N_{B}} \frac{\beta}{\left|\mathbf{r}_{m}-\mathbf{r}_{n}\right|},
\end{aligned}
$$

where we defined the charge ratio parameter $\beta=Q_{B} / Q_{A}$ and expressed the coordinate and energy, respectively, in the following units: $r_{0}=\left(2 Q_{A}^{2} / m \epsilon_{0} \omega_{0}\right)^{1 / 3}$ and $E_{0}=$ $\left(m \omega_{0}^{2} Q_{A}{ }^{4} / 2 \epsilon_{0}{ }^{2}\right)^{1 / 3}$. All our numerical results will be given in these units.

To obtain the stable configurations we used the Monte Carlo simulation technique supplemented by the modified Newton method in order to speed up the computation and to increase the accuracy of the found energy values (see Ref. [26] for details). By starting from a large number of different random initial configurations we are confident that we find the GS configuration. Depending on the values of the total number of particles and on the charge ratio parameter, between several hundred to several thousand random initial configurations were generated. Such a computational procedure ensures that the GS configuration is obtained for values of $\beta \lesssim 0.85$, since for larger values of $\beta$, i.e., for $0.85 \lesssim \beta \lesssim 1.00$, a vitric phase was found for most of the values of $N$. The latter phase is characterized by an impressive number of meta-stable states caused by the close proximity of the charges of the different types of particles. Fortunately, within the vitric phase most of the clusters' energies are very close to each other with configurations that are all very similar and, consecutively, their mechanical stability have similar properties. In practice, for values of $\beta \gtrsim 0.85$, due to the large number of simulations performed for the same parameters, we are confident to obtain the ground state or at worst a configuration very close to that of the ground state.

The systems treated in this work are not large enough for us to employ thermodynamics. In fact, the largest system treated in this work has $N=38$ particles. Under these conditions the temperature actually corresponds to an average kinetic energy. The systems chosen are small, while large systems do not show properties of magic clusters. As stated in the Introduction, only the systems with $N=6,12,13$, and 38 are magic clusters. Thus terms like "melting temperature" or "critical temperatures" used in this article are employed in the context of small systems and not in the context of systems in the thermodynamic limit.

The simulation methodology used to obtain the melting temperature consists of three main stages: (1) the velocities of the particles are scaled for the purpose of increasing the temperature, (2) the system is allowed to evolve during a few steps in order to allow it to relax, and finally (3) we perform the desired measurements. After finalizing this latter, the cycle restarts for the system at a higher temperature. These three stages are performed successively until the system melts completely. In the first stage we employ the velocity scaling scheme, while in the last two we employ the usual velocity Verlet algorithm.

The critical temperature for angular melting can be determined by computing the mean-squared angular displacement $[25,30]$. For a 3D system the expression for the meansquared angular displacement becomes

$$
\Delta \alpha_{\gamma}=\frac{1}{N} \sum_{i=1}^{N_{\gamma}}\left\langle\alpha_{i j}^{2}\right\rangle-\left\langle\alpha_{i j}\right\rangle^{2},
$$

where $\alpha_{i j}$ is the angle between the position vectors of two neighboring particles $i$ and $j$. In this equation, $N$ indicates the 


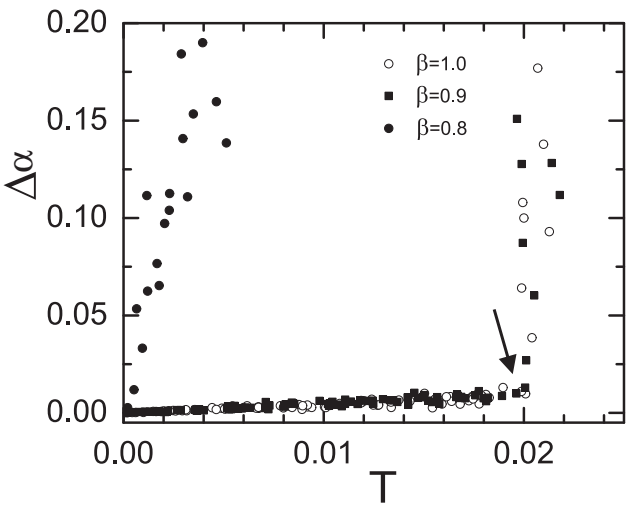

FIG. 1. Angular mean-square displacement as a function of temperature for the system with $N=12$ and $N_{B}=3$, and different values of $\beta$.

total number of particles, and $\langle\cdots\rangle$ is an average over time. In order to characterize the melting temperature, we made use of a Lindemann-like criterion. Close to the melting process, the angular averaged displacement starts to deviate rapidly from its low temperature linear dependence [25], which one determines the melting temperature.

\section{SMALL SIZE SYSTEMS}

Figure 1 shows the mean-squared angular displacement as a function of temperature for the system with $N=12$ particles, $N_{B}=2$ and the following three values of the charge ratio parameter: $\beta=1.0$ (opened circles), 0.9 (black squares), and 0.8 (closed circles). Previously, we found that for the case of isotropic particles the $N=12$ cluster is a magic cluster. As we have seen in the latter section, by definition, the angular melting temperature is the one where the averaged angular displacement loses its linear dependence with the temperature. Using this criterion, one finds that the angular melting temperatures, for the systems with $\beta=1.0$ and 0.9 , are approximately the same, i.e., $T_{c}=0.201$ (see Fig. 1, downward black arrow). This fact shows that the system with $\beta=0.9$ also exhibits magic cluster properties, since its mechanical stability is comparable to that of the system with $\beta=1.0$. In contrast, we notice from Fig. 1 that the system with $\beta=0.8$ is mechanically much less stable, since it melts at a very low temperature, i.e., $T_{c} \simeq 0.002$.

From the latter paragraph, it becomes evident that the mechanical stability is strongly influenced by the value of the charge ratio parameter. In order to understand the reason, we will look in detail to how the cluster symmetry depends on the parameter $\beta$. Figure 2(a) shows the radial position of the particles $r$ as a function of $\beta$ for the system with $N=12$ and $N_{B}=3$. The radial positions of the $A$ and $B$ type particles are indicated, respectively, by black and red (gray) lines. Notice that, for $\beta=1.0$, i.e., for the monodisperse case, all particles have the same radius $r=1.27$, and that the overall structure is an icosahedron, which is the only single shell structure forming a magic cluster. A schematic representation of this GS configuration is displayed in Fig. 3(a), where black and red (gray) balls represent, respectively, particles of the $A$ and $B$ types. In this latter figure, bonds between the different

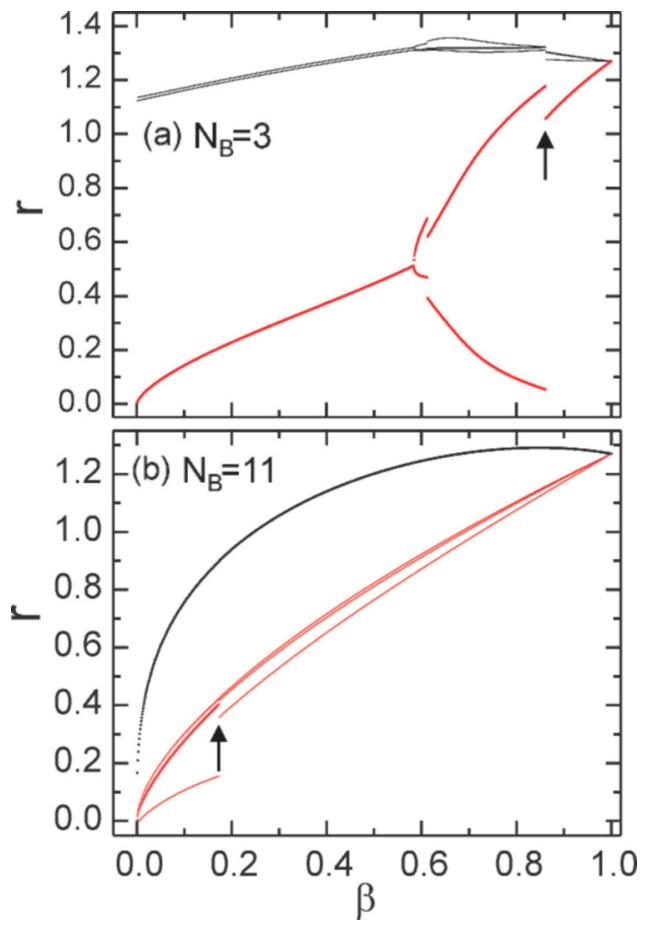

FIG. 2. (Color online) Zero-temperature particles position $r$ as a function of the charge ratio parameter $\beta$ for the system with $N=12$ for (a) $N_{B}=3$ and (b) $N_{B}=11$. Black and red (light gray) symbols correspond to particles of the $A$ and $B$ type, respectively.

balls are drawn only to enhance visualization. We can see from Fig. 2(a) that even a small decrease of $\beta$ leads to an immediate reduction of the radial positions of the $B$ type particles. However, notice that for large values of $\beta$ the system still has an icosahedral symmetry [see, for instance, Fig. 3(b) for $\beta=0.9]$. The reason is that the particles segregation evolves continuously, i.e., without the mediation of first-order

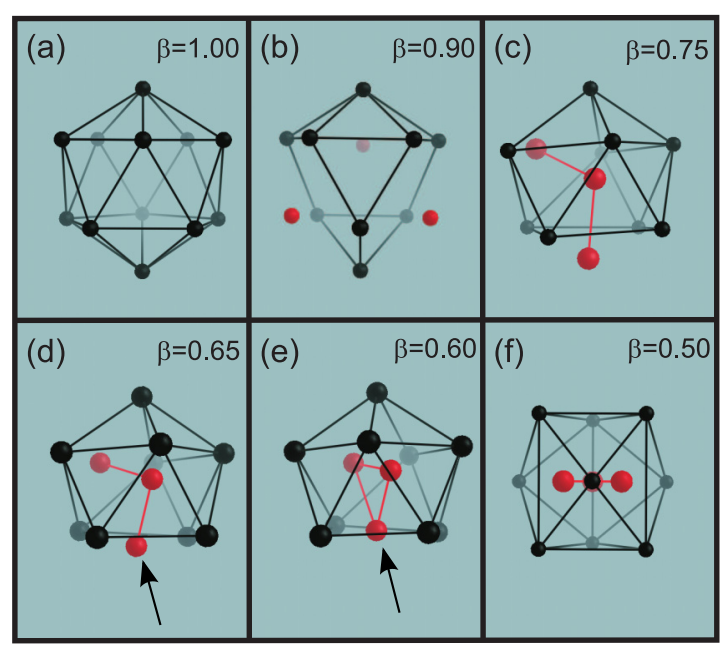

FIG. 3. (Color online) Schematic representation of the GS configuration for a system with $N=12$ particles, $N_{B}=3$, and several values of the charge ration parameter, $\beta$, as indicated. Particles of the type $A(B)$ are represented by black [red (light gray)] full balls and lines joining them are used only to enhance visualization. 


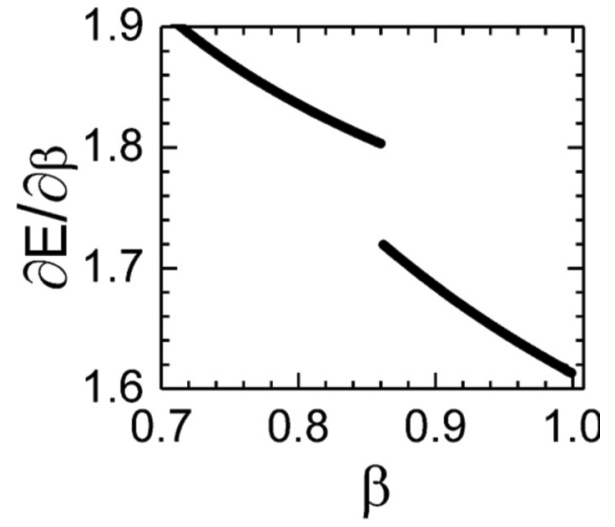

FIG. 4. First derivative of the energy, $E$, with respect to the charge ratio parameter, $\beta$, for the system with $N=12$ and $N_{B}=3$.

structural phase transitions [24,26], at least for values of $\beta$ relatively large.

When further decreasing $\beta$, the radial position of the particles pass through an abrupt change at $\beta=0.865$ [see Fig. 2(a), upward arrow], which is characteristic of a structural phase transition of the first order, as indicated by the discontinuity of the first derivative of the energy, $E$, with respect to the parameter $\beta$ (see Fig. 4). Figure 3(c) shows the GS configuration for a value of $\beta$ slightly smaller than this critical one, i.e., for $\beta=0.75$. We can see that this GS configuration has 11 particles in the outer shell, while the 12th particle sits in the center of the cluster. Thus the dramatic decrease of the mechanical stability of the system, as demonstrated in Fig. 1, occurs when the system loses its icosahedral symmetry characteristic of a monodisperse system having 12 particles.

Figure 5 presents the angular melting temperature, $T_{c}$, for the system with $N=12$ particles and different values of $N_{B}$. For small values of $N_{B}$, i.e., $N_{B}<5$ [see Fig. 5(a)], and large values of the charge ratio parameter, i.e., $\beta>0.8$, the melting temperature is relatively large, i.e., $T_{c} \geqslant 0.02$. But for $\beta<0.8$, the melting temperature drops to a very low value, i.e., $T_{c}<0.008$. Therefore, we may conclude that that occurs before the first-order structural phase transition, i.e., for $\beta>\beta_{c}$, is not significatively strong in order to destroy the magic cluster properties of the system, at least as long as the total number of particles of the $B$ type is small. Moreover, for systems with small values of $N_{B}$, the critical point $\beta_{c}$ separates two distinct regimes, i.e., the magic from the normal cluster regime.

The transition magic-to-normal cluster is not always as sharp when varying the $\beta$ parameter as we found for systems with small $N_{B}$. In fact, as the value of $N_{B}$ increases the magicto-normal cluster transition becomes more gradual as we can see by contrasting Figs. 5(b) and 5(c) with Fig. 5(a). There are two main reasons for this: (1) because of the lack of a first-order structural phase transition and (2) because the $A-B$ particle segregation as a consequence of decreasing $\beta$ does not lead to an abrupt change of the icosahedral cluster symmetry. For instance, Fig. 2(b) shows that for the system with $N=12$ and $N_{B}=11$ the particle segregation evolves continuously down to the critical value $\beta_{c}=0.175$ [see upward black arrow in Fig. 2(b)], at which the system suffers a structural firstorder phase transition. Therefore, we can conclude that the

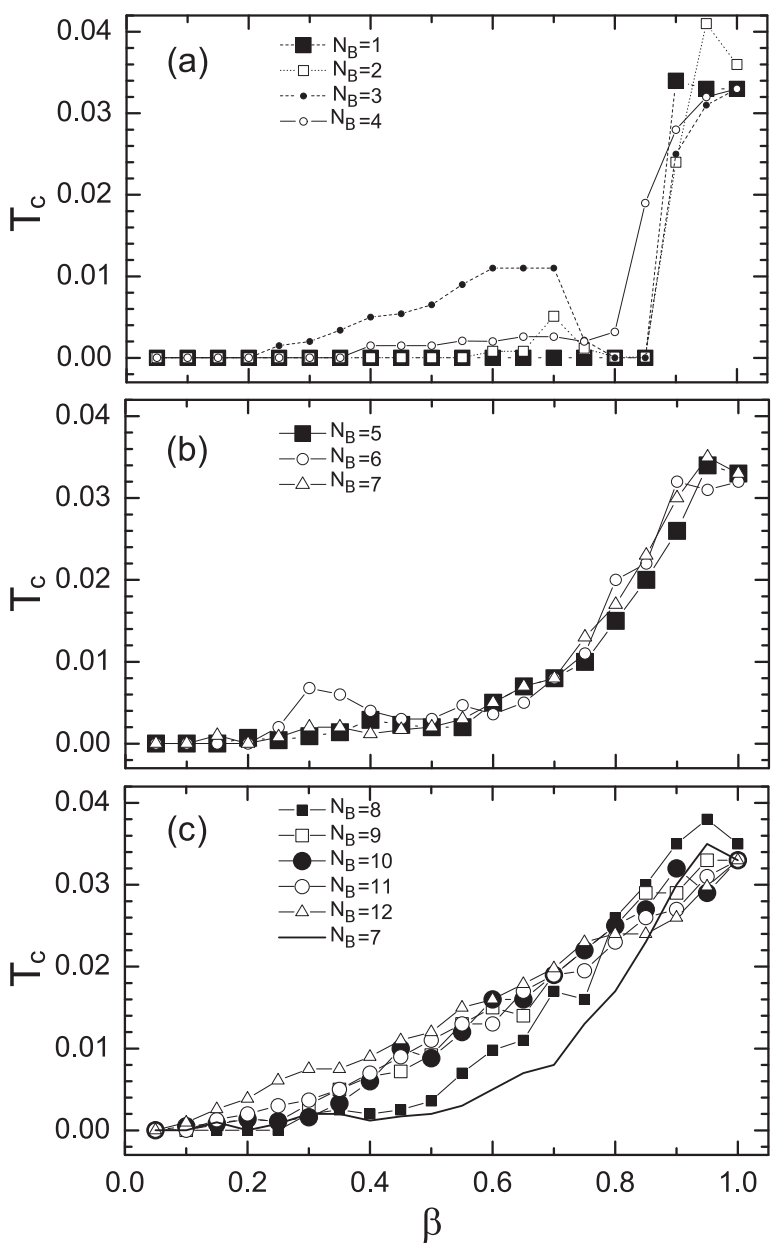

FIG. 5. Angular melting temperature, $T_{c}$, as a function of the charge ratio parameter, $\beta$, for different number of $B$-type particles, $N_{B}$, and fixed total number of particles $N=12$.

transition magic-to-normal cluster, for systems with large and intermediate numbers of particles of the $B$ type, evolves as a function of $\beta$ gradually, as we found for systems with small values of $N_{B}$.

Notice that a direct investigation of the mechanical stability of the system can reveal new features not uncovered in Ref. [27]. For instance, Fig. 5(a) shows that the system with $N_{B}=3$ exhibits an anomalous behavior, i.e., a reentrant behavior in the value of the melting temperature. A large mechanical stability is achieved for $0.60<\beta<0.70$ which is due to the fact that one of the particles of type $B$, which is indicated by a black arrow, in Figs. 3(d) and 3(e), becomes laterally confined by a ring containing four particles of type $A$. Notice that these structures form a hybrid configuration, composed of a 3D top part which is reminiscent of an icosahedral structure and a 2D bottom part which forms a ring structure. Finally, we see from Fig. 3(f) that the GS configuration for $\beta=0.50$ corresponds to a multiple ring structure. Therefore, this result demonstrates two facts: (1) that binary systems are able to form multiple ring structures and (2) that similar to the multiple rings of monodisperse systems highly symmetrical structures for binary systems also result in a large melting temperature. 


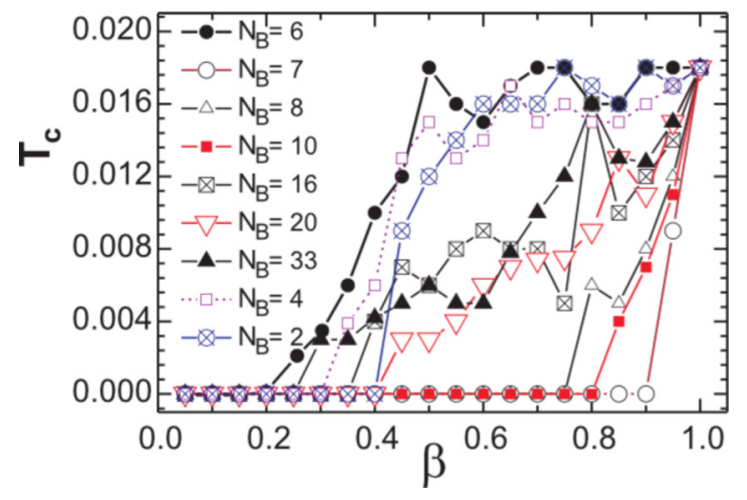

FIG. 6. (Color online) Angular critical melting temperature, computed for the 32 most external particles, as a function of the charge ratio parameter for the system with 38 particles, and different values of $N_{B}$.

\section{LARGE SIZE SYSTEMS}

Previously we found in Refs. [24,25] that the only magic cluster of large systems, i.e., clusters with two shells, is the GS configuration of the system with $N=38$ particles. Additionally, it was also shown that the magic cluster properties are related with a specific closure condition satisfied by the particles of the external shell, i.e., the 12 five coordinated particles form an icosahedron, while each of the 20 six coordinated particles are found in the center of one of the icosahedral faces (see Fig. 4 in Ref. [25]). Such a highsymmetry configuration leads to a high melting temperature, which is not seen in other size systems (see Refs. [24,25] for more detail).

In this section, we investigate how the properties of large magic binary clusters are influenced by the relative number of different particles and different charges. We start by presenting (see Fig. 6) the angular melting temperature, $T_{c}$, calculated for the group of 32 particles farthest from the center of the cluster, that is, the 32 particles with the largest radial distance from the center. From Fig. 6, we notice that the dependence of $T_{c}$ on $\beta$ strongly depends on the number of particles of type $B$. As $\beta$ decreases, the critical temperature also decreases until it reaches a value close to zero. We can identify three regimes for this two shell system, which differ in the way that the critical temperature goes to zero. The mechanical stability of the systems with $N=2,4$, and 6 particles possess the same characteristics. In fact, as seen from Fig. 6, the clusters' melting temperatures stay high until about $\beta=0.4$. When further decreasing $\beta$, we see a rapid drop in $T_{c}$ reaching almost zero. For didactic purposes we assign systems with $N=2$, 4 , and 6 to belong to group I. For the systems with $N=7$, 8 , and $10 T_{c}$ also decreases rapidly. For such systems, the melting temperature reaches zero already for $\beta=0.75$. We will say that these clusters belong to group III. Finally, we draw attention to a group of configurations in which $T_{c}$ is not reduced as slowly as in group I nor as fast as in group III. Such configurations are represented here (see Fig. 6) by the systems with $N=16,20$, and 33 . Nevertheless, note that for these configurations belonging to group II, $T_{c}$ reaches zero for $\beta \leqslant 0.35$.
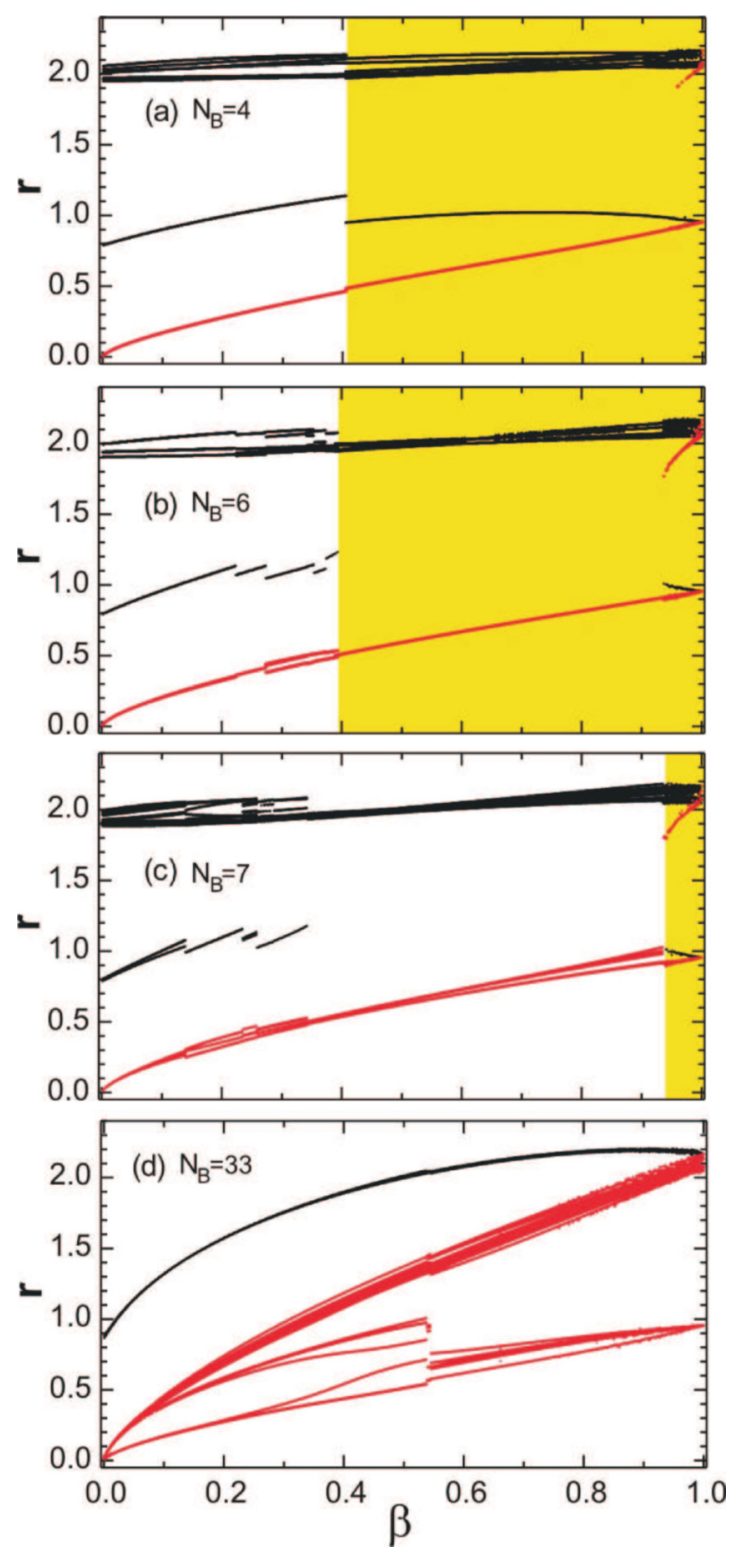

FIG. 7. (Color online) Radial position of the particles in the cluster as function of the charge ratio parameter $\beta$ for the system with $N=38$ particles and several different values of $N_{B}$. The yellow (light gray) region in figures (a), (b), and (c) indicate the $\beta$ interval over which the external shell of the clusters contains 32 particles. The $A$ and $B$ type of particles are indicated by black and red (light gray) curves.

Next we will show how the mechanical stability of the systems belonging to the different groups are related to the radial distribution of particles. To this end, Fig. 7 shows the radial position of the particles as a function of $\beta$ for systems with a total number of particles equal to 38 and different values of $N_{B}$ (particles of types $A$ and $B$ are shown respectively by curves in black and red). The yellow area in Fig. 7(a) for $N_{B}=4$ shows the range in $\beta$ for which the external shell is still populated by 32 particles. This region extends from the value of $\beta=1.0$ down to 0.405 , where the system undergoes a first-order transition at which the inner shell splits into two sets of particles, i.e., an inner set containing two particles of 
type $B$ (red curve) and a second set containing four particles of type $A$ (black curve just above the red one). Right after this transition one particle of type $A$ leaves the outermost shell to the innermost, so that the first and second shells contain, respectively, 31 and 5 particles of type $A$. Such a change in the number of particles in the outer shell leads to a significant reduction in the melting temperature, as seen in Fig. 6, where the value of $T_{c}$ drops sharply.

The mechanical stability of the system with $N_{B}=6$ particles behaves similar to that of the system with $N_{B}=4$ particles. Figure 7(b) shows that the yellow region extends from $\beta=1.0$ to 0.395 , and that immediately outside this region an intermediate shell containing two particles of type $A$ emerges. Thus the number of particles in the outer shell is reduced to 30 . We also observed that around the critical charge ratio parameter $\beta=0.395$ the outer shell loses its mechanical stability and, consequently, the melting temperature decreases dramatically (see Fig. 6).

Therefore, by comparing the results of Figs. 6 and 7 for the $N_{B}=4$ and 6 cases, respectively, we can conclude that the mechanical stability of the cluster remains high as long as the outer shell has 32 particles. It is also important to observe that the inner shell does not exert an important role in the stability of the outer shell. For example, for the $N_{B}=4$ case [Fig. 7(a)], although the inner shell is divided into two sets the melting temperature does not change significantly. Likewise, for the $N_{B}=6$ case, the melting temperature also does not decrease significantly even for a reduction of the radius of the inner subcluster formed by particles of the type $B$ [see Fig. 7(b)].

However, for the system with $N_{B}=7$ the loss of stability occurs for much larger values of $\beta$ as can be seen from Figs. 7(c) and 6. The interval for which the outer shell has 32 particles (see yellow region) is very narrow, that is, $0.945<\beta<1.0$. We have found that a system with $N_{B}=8$ and 10 can hold 32 particles in its outer shell only within a very narrow range of the charge ratio parameter, that is, from $\beta=1.00$ down to $\beta=0.83$ and 0.89 , respectively. Notice that in all those cases the values of $N_{B}$ are only slightly larger than 6 , that is, the number of particles of the internal shell for the monodisperse case. This explains the rapid decrease of $T_{c}$, as the charge ratio parameter is reduced below 1 (see Fig. 6) for the systems with $N_{B}=7,8$, and 10. In other words, systems with value of $N_{B}$ only slightly larger than 6 pass through a structural first-order phase transition for larger values of $\beta$, resulting in a reduction of the number of particles in the external shell. Ultimately, such a fact leads to an abrupt decrease of the angular melting temperature occurring at larger values of the charge ratio parameter, e.g., $\beta_{c} \simeq 0.85$.

Finally, note that for the system $N_{B}=33$, the $B$-type particles gradually move towards the interior of the cluster, that is, without any abrupt first-order phase transition. The displacement occurs gradually over a broad range of $\beta$, that is, $0.45<\beta<1.0$ [see Fig. 7(d)], and during this interval the symmetry of the system is only slightly changed. This situation is different from that seen in group III, which is mediated by a first-order transition. For the $N_{B}=33$ case, the melting temperature takes intermediate values between those seen in groups I and III. We found that the other systems belonging to group II, such as those with $N_{B}=16$ and 20, have intermediate melting temperatures due to the same kind of gradual shift seen for the system with $N_{B}=33$ particles.

\section{CONCLUSIONS}

In this paper we investigated the disappearance of angular order in isotropically confined three-dimensional binary systems. The mechanical properties of such systems were investigated by molecular-dynamic simulations and the angular melting temperatures were calculated by using a Lindemanntype criterion. Our research focused on revealing the main characteristics involving structural transitions between magic and normal clusters due to the reduction in the charge ratio parameter $\beta$. We consider representative systems consisting of $N=12$ and 38 particles that form monodisperse magic clusters, respectively, with one and two shells.

The transition from magic to normal cluster is induced by reducing $\beta$ and was found to have two distinct regimes when the system has only one shell. The first regime occurs when the number of particles of type $B$ is relatively small, for example, for $N_{B}$ values between 1 and 4 . In this situation the particles of type $B$ can leave the outer shell even for large values of $\beta$. We note that such structural changes are mediated by a first-order phase transition. On the other hand, systems containing a relatively large number of particles of type $B$ behave differently. For example, for the case $N_{B}=9$, we found that with decreasing $\beta$, the transition from magic to normal cluster occurs gradually, which leads to a gradual decrease of the melting temperature instead of an abrupt one.

As a two shell system we considered the mechanical stability of the system with 38 particles for various values of $N_{B}$. The GS of the monodisperse system with $N=38$ particles constitute the only two shell configuration that is a magic cluster. Such a system has 6 particles in the inner shell and 32 in the outer one. We found that the mechanical stability of such a system has three distinct regimes whose characteristics depend on the difference between $N_{B}$ and the total number of particles in the internal shell, that is, 6 particles. For group I the number of particles of type $B$ is less than or equal to 6 . With decreasing $\beta$, particles of type $B$ migrate to the interior of the cluster, which eventually led to the reduction of the innermost shell of the system. More importantly, we found that such displacement of particles does not affect the melting temperature until a first-order phase transition occurs. After this transition the outer shell contains less than 32 particles.

Group III are formed by those systems in which the number of particles of type $B$ is slightly higher than the number of particles in the inner shell, that is, slightly larger than 6 . Examples investigated in this article comprise the cases $N_{B}=7,8$, and 10 . As the $\beta$ parameter decreases we found that the $B$ type particles, which are initially in the external shell, jump to the inner shell. This occurs at a relatively high critical value of $\beta$, i.e., around $\beta=0.8$. At this value the melting temperature of the system drops drastically due to this reduction of the number of particles in the outer shell. On the other hand, for a system belonging to the group II we verified that the melting temperature drops more gently than for group I but faster than for group III. This fact occurs because the number of particles of type $B$ in the external 
shell is relatively high. For example, in the case $N_{B}=33$, which was also investigated in this article, the number of particles in the internal shell is 27 . Thus, as $\beta$ decreases, a relatively high number of $B$ type particles move to the inner region of the cluster, which contributes to maintaining the symmetry of the system. Moreover, such displacement of type $B$ particles evolves continuously, i.e., even without a second-order transition, which occurs only for relatively low values of $\beta$, that is, $\beta \simeq 0.55$.

Finally, we note that binary systems can also self-organize into multiple ring structures, as we have seen, for example, for the cases $N=12, N_{B}=3$, and $\beta=0.5$. Multiple ring structures were previously found only in anisotropic systems [28,29], and they were found to possess a high melting temperature. Here we demonstrated that high-symmetry configurations can also exhibit a large melting temperature.

\section{ACKNOWLEDGMENTS}

This work was supported by FACEPE (Fundação de Amparo à Ciência e Tecnologia do Estado de Pernambuco) Grant No. APQ-1800-1.05/12, the bilatera project between $\mathrm{CNPq}$ and FWO-VL, and the Flemish Science Foundation (FWO-V1).
[1] D. J. Wineland, J. C. Bergquist, W. M. Itano, J. J. Bollinger, and C. H. Manney, Phys. Rev. Lett. 59, 2935 (1987).

[2] M. Drewsen, C. Brodersen, L. Hornekaer, J. S. Hangst, and J. P. Schiffer, Phys. Rev. Lett. 81, 2878 (1998).

[3] A. V. Filinov, M. Bonitz, and Y. E. Lozovik, Phys. Rev. Lett. 86, 3851 (2001).

[4] C. C. Grimes and G. Adams, Phys. Rev. Lett. 42, 795 (1979).

[5] H. Löwen, Phys. Rep. 237, 249 (1994).

[6] G. E. Morfill and A. V. Ivlev, Rev. Mod. Phys. 81, 1353 (2009).

[7] M. Bonitz, C. Henning, and D. Block, Rep. Prog. Phys. 73, 066501 (2010).

[8] G. Blatter, M. V. Feigel'man, V. B. Geshkenbein, A. I. Larkin, and V. M. Vinokur, Rev. Mod. Phys. 66, 1125 (1994).

[9] A. K. Geim, I. V. Grigorieva, S. V. Dubonos, J. G. S. Lok, J. C. Maan, A. E. Filippov, and F. M. Peeters, Nature (London) 390, 259 (1997).

[10] J. E. Hug, F. van Swol, and C. F. Zukoski, Langmuir 11, 111 (1995).

[11] S. Neser, T. Palberg, C. Blechinger, and P. Leiderer, Prog. Colloid Polym. Sci. 104, 194 (1997).

[12] A. Melzer, A. Homann, and A. Piel, Phys. Rev. E 53, 2757 (1996).

[13] H. Thomas and G. E. Morfill, Nature (London) 379, 806 (1996).

[14] L. I, W.-T. Juan, C.-H. Chiang, and J. H. Chu, Science 272, 1626 (1996).

[15] A. Piel and A. Melzer, Plasma Phys. Control. Fusion 44, R1 (2002).

[16] G. E. Morfill, H. M. Thomas, U. Konopka, H. Rothermel, M. Zuzic, A. Ivlev, and J. Goree, Phys. Rev. Lett. 83, 1598 (1999).
[17] A. P. Nefedov, G. E. Morfill, V. E. Fortov, H. M. Thomas, H. Rothermel, T. Hagl, A. V. Ivlev, M. Zuzic, B. A. Klumov, A. M. Lipaev, V. I. Molotkov, O. F. Petrov, Y. P. Gidzenko, S. K. Krikalev, W. Shepherd, A. I. Ivanov, M. Roth, H. Binnenbruck, J. A. Goree, and Y. P. Semenov, New J. Phys. 5, 33 (2003).

[18] M. Klindworth, A. Piel, A. Melzer, U. Konopka, H. Rothermel, K. Tarantik, and G. E. Morfill, Phys. Rev. Lett. 93, 195002 (2004).

[19] M. Klindworth, O. Arp, and A. Piel, J. Phys. D: Appl. Phys. 39, 1095 (2006).

[20] O. Arp, D. Block, A. Piel, and A. Melzer, Phys. Rev. Lett. 93, 165004 (2004).

[21] M. Bonitz, D. Block, O. Arp, V. Golubnychiy, H. Baumgartner, P. Ludwig, A. Piel, and A. Filinov, Phys. Rev. Lett. 96, 075001 (2006).

[22] P. Ludwig, S. Kosse, and M. Bonitz, Phys. Rev. E 71, 046403 (2005).

[23] V. Golubnychiy, H. Baumgartner, M. Bonitz, A. Filinov, and H. Fehske, J. Phys. A: Math. Gen. 39, 4527 (2006).

[24] S. W. S. Apolinario, B. Partoens, and F. M. Peeters, New J. Phys. 9, 283 (2007).

[25] S. W. S. Apolinario and F. M. Peeters, Phys. Rev. E 76, 031107 (2007).

[26] V. A. Schweigert and F. M. Peeters, Phys. Rev. B 51, 7700 (1995).

[27] S. W. S. Apolinario and F. M. Peeters, Phys. Rev. E 83, 041136 (2011).

[28] S. W. S. Apolinario, B. Partoens, and F. M. Peeters, Phys. Rev. B 77, 035321 (2008).

[29] S. W. S. Apolinario and F. M. Peeters, Phys. Rev. B 78, 024202 (2008).

[30] V. M. Bedanov, G. V. Gadiyak, and Y. E. Lozovik, Phys. Lett. 109, 289 (1985). 